\title{
Pituitary Adenoma with Apoplexy with Arteriovenous Malformation
}

\author{
Rajeev Bansal ${ }^{1}$ Jitendra Shekhawat ${ }^{1}$ Devendra Purohit ${ }^{1}$ \\ ${ }^{1}$ Department of Neurosurgery, SMS Medical College and Hospitals, \\ Jaipur, Rajasthan, India \\ Address for correspondence Rajeev Bansal, MBBS, MS, MCh, \\ Department of Neurosurgery, SMS Medical College and Hospitals, Jaipur, \\ Indian J Neurosurg 2017;6:59-61. \\ Rajasthan 302004, India (e-mail: rajeev_bansal234@yahoo.co.in).
}

\begin{abstract}
Keywords

- arteriovenous malformation

- pituitary adenoma

- primary brain tumor

Background This is a rare association of pituitary adenoma with apoplexy with arteriovenous malformation as only single case was reported prior to this case as per our knowledge.

Case Description A 25-year-old man presented with chief complaints of headache of sudden onset, severe in intensity with sudden loss of vision. Imaging shows pituitary adenoma with apoplexy with right parietal arteriovenous malformation. The patient was managed with embolization for parietal arteriovenous malformation and medically for pituitary adenoma with apoplexy.

Conclusion Pituitary apoplexy can be treated conservatively if no features of mass effect present. Follow-up of the patient must be done regularly to look for the size of pituitary adenoma and recurrence of arteriovenous malformation.
\end{abstract}

\section{Case Report}

A 25-year-old man admitted to our department with chief complaints of headache and giddiness for last 2 weeks. Two weeks back the patient had history of headache of sudden onset, severe in intensity with sudden loss of vision. He regained his vision without any deficit within minutes. There was no history of unconsciousness, seizures or focal deficit, and persistent diminution of vision. On examination the patient had visual acuity $6 / 6$ in the both eyes with no field defects. On fundus examination no abnormality was detected. Hormone analysis showed level of prolactin greater than $200 \mathrm{ng} / \mathrm{mL}$.

Contrast-enhanced computed tomography (CECT) of the brain (- Figs. $\mathbf{1}$ and $\mathbf{2}$ ) on admission showed welldefined, contrast-enhancing, hyperdense lesion of size $24 \times 20 \times 17 \mathrm{~mm}$. Lesion seen in parafalcine region of the right parietal lobe associated with prominent perilesional vessels appeared draining in superior saggital sinus suggestive of arteriovenous malformation (AVM). There is evidence of widening of sella with presence of soft tissue density lesion of size $20 \times 17 \times 20 \mathrm{~mm}$ pituitary adenoma.

received

February 24, 2016

accepted

April 18, 2016

published online

March 16, 2017
DOI http://dx.doi.org/

10.1055/s-0036-1584601. ISSN 2277-954X.
Magnetic resonance imaging (MRI) of the brain and sella showed a mass lesion in sella showing presence of solid and cystic component. The cystic component appeared hyperintense on $\mathrm{T} 2$ whereas solid component appeared isointense to gray matter on $\mathrm{T} 1$ and $\mathrm{T} 2 \mathrm{~W}$ images. Hemorrhage was noted within lesion showing fluid level and hypointense on T2W images. Mass was seen extending into the sphenoid sinus. A small AVM was noted along medial aspect of right parieto-occipital lobe showing presence of multiple vascular channels and early draining vein into the superior saggital sinus and into deep venous sinuses. There was associated mild perilesional gliosis. It showed heterogenous enhancement on postcontrast enhancement.

Cerebral CT angiography showed AVM with nidus measuring $25 \times 22 \mathrm{~mm}$ in parafalcine region of the right high parietal lobe with the caliber of draining vein measuring $4 \mathrm{~mm}$ draining into superior saggital sinus. The AVM is supplied by the right callosomarginal artery, branch of right anterior cerebral artery.

Cerebral digital subtraction angiography (-Fig. 3) showed AVM of size $7.95 \times 16.13 \mathrm{~mm}$ of the right parietal lobe supplied by the right pericallosal artery (branch of right

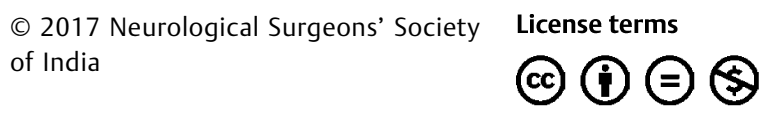




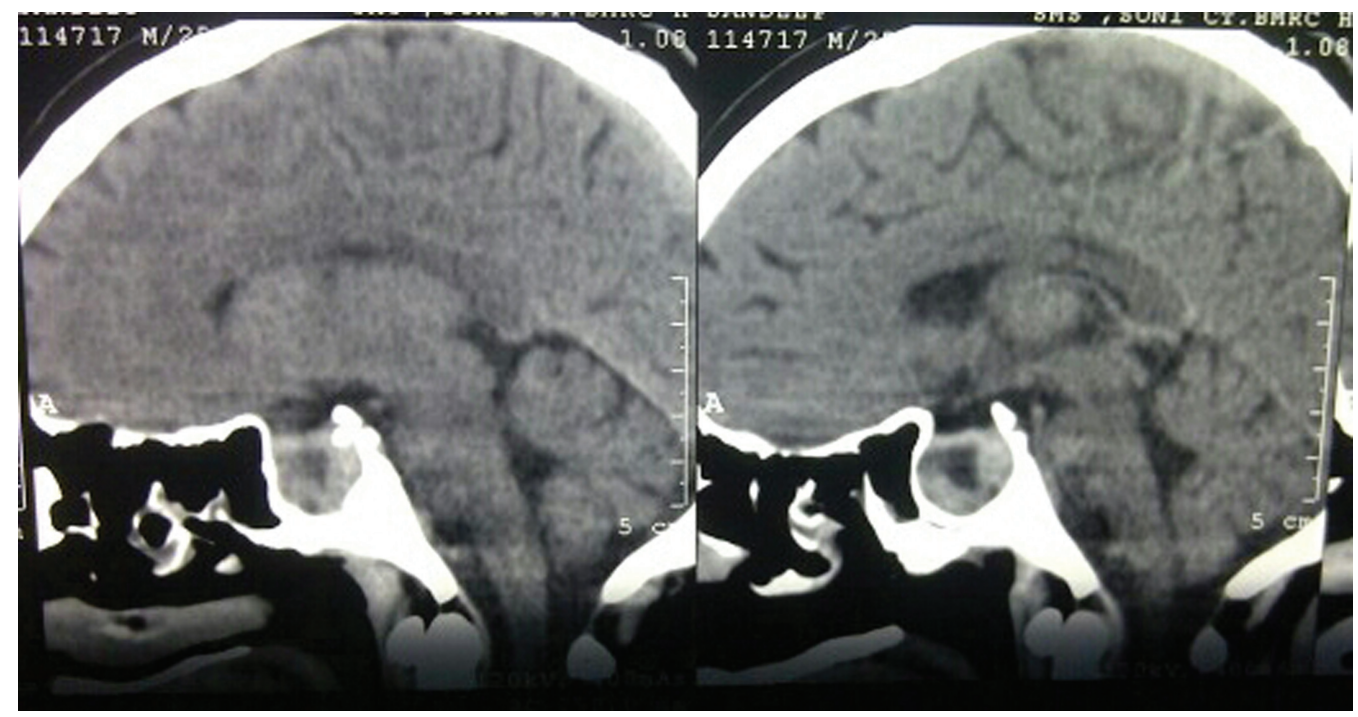

Fig. 1 CECT of the brain saggital cuts showing right parietal arteriovenous malformation and pituitary adenoma.

anterior cerebral artery) and right posterior cerebral artery. It was draining into superficial venous system. Embolization with the help of glue was done for parietal AVM. Pituitary adenoma with apoplexy was managed medically with dopamine agonist as the patient did not have vision loss or signs of mass effect. The patient responded well to treatment.

\section{Discussion}

The association of a primary brain tumor with intracranial AVM is uncommon. When accompanied by intracranial $\mathrm{AVM}$, a primary brain tumor is usually a glioma. A pituitary adenoma is rarely associated with intracranial
AVM. Licata et $\mathrm{al}^{1}$ reported only one case of association of pituitary adenoma with intracranial AVM.

There have been 74 reported cases of brain tumors associated with AVM to the best of our knowledge. The rate of this rare association was reported to be $0.1 \%$. Concerning the histology of the brain tumors involved in these cases, there were 12 cases of pilocytic astrocytoma, 10 of astrocytoma, 6 of malignant astrocytoma, 20 of oligodendroglioma, 3 of glioblastoma, 8 of meningioma, 5 of acoustic tumors, and 3 of pleomorphic xanthoastrocytoma. Other tumors found in association with AVM include hemangioblastoma, hemangiopericytoma, ganglioneuroma, subependymal giant astrocytomas, and craniopharyngioma. In the remaining two cases, histology was simply described as glioma. Thus

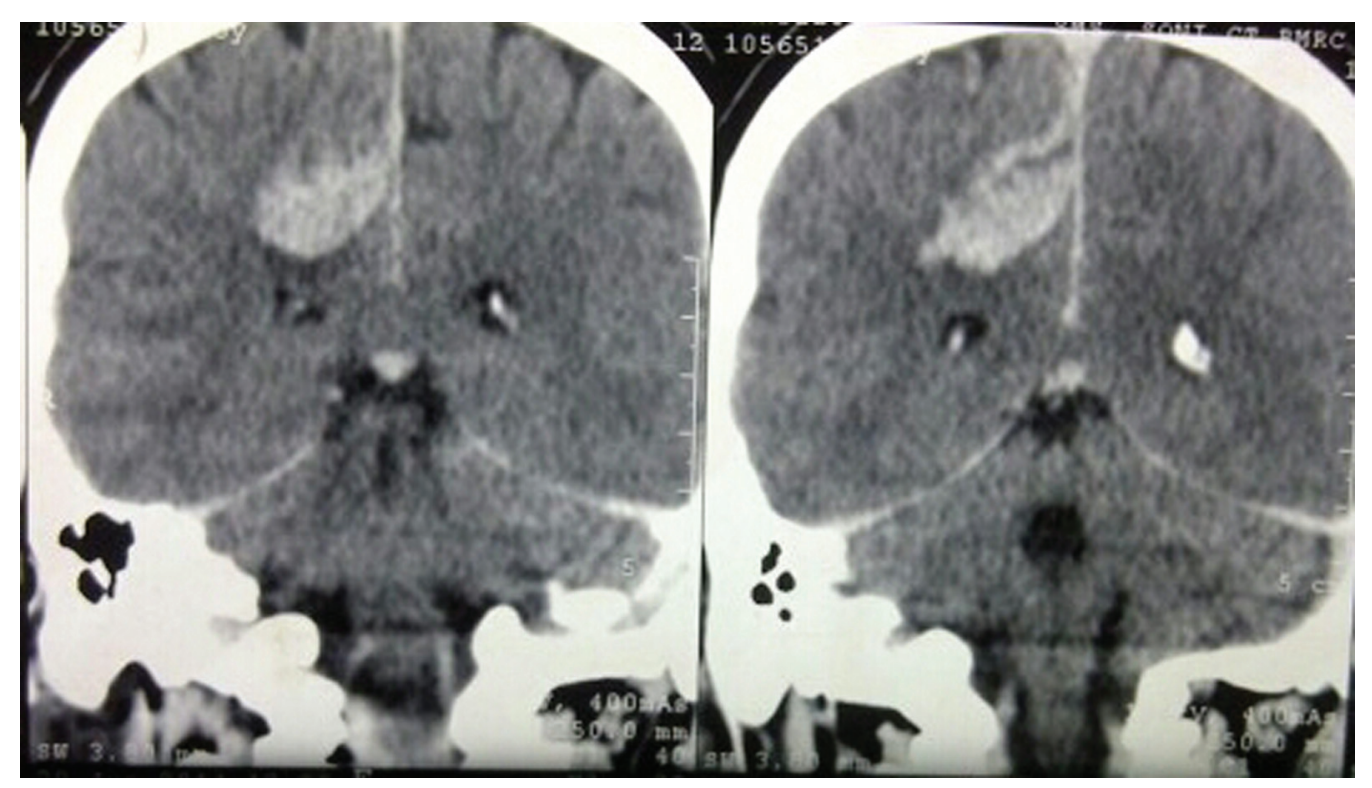

Fig. 2 CECT of the brain coronal cuts showing right parietal arteriovenous malformation and pituitary adenoma. 


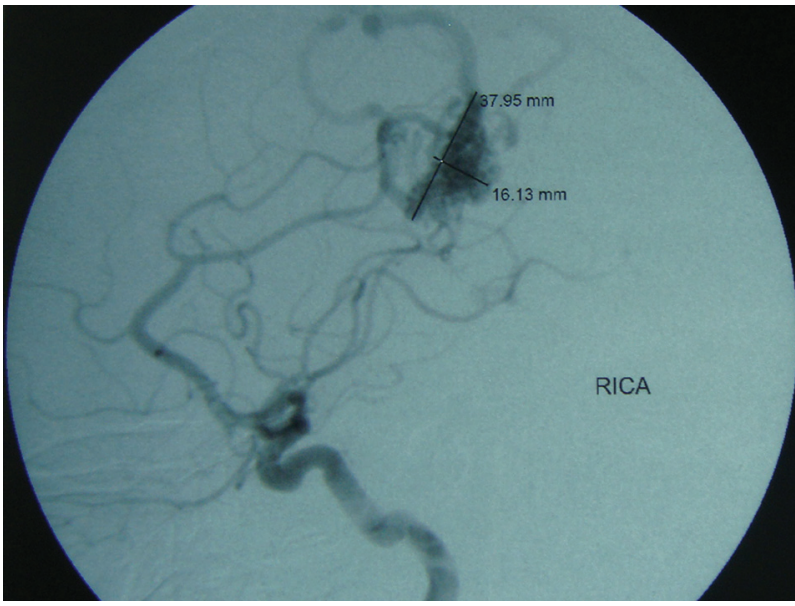

Fig. 3 Cerebral digital subtraction angiography showing right parietal arteriovenous malformation supplied by right pericallosal and right splenial artery and drained into superficial venous system.

anaplastic oligodendroglioma is the type of tumor most frequently associated with AVM. ${ }^{2}$

Concerning the positional relation between the tumor and AVM, Bitoh et al divided the association into three types. In type 1, two lesions coexist in different regions, even in different cerebral lobes or in different hemispheres. In type 2 , two lesions are contiguous or completely intermixed. In type3, two lesions are adjacent but completely separated. ${ }^{3}$ There are different explanations for the pathogenesis of the two lesions. ${ }^{3}$ In Bitoh type 1, two lesions are in different regions and showed different pathologic characteristics. The coexistence of the two lesions is purely by chance. In Bitoh type 2 or 3, it is indicated that the formation and growth of one lesion is affected by other lesion, or the two lesions are both affected by another factor. Some hypotheses are as follows:

- AVM induces a tumor.

- Tumor causes AVM.

- Tumor and AVM are both caused by a virus.

- Tumor and AVM are both related to a genetic factor.

- Two lesions are derived from different embryo remnant tissues.

Our case falls into Bitoh type 1 because AVM was located in the right parietal lobe without direct relation to pituitary adenoma.

\section{Take-Home Messages}

- Pituitary apoplexy can present without any complication.

- Pituitary apoplexy can be treated conservatively if no features of mass effect present.

- Follow-up of the patient must be done regularly to look for the size of pituitary adenoma and recurrence of AVM.

- AVM in a young patient should be treated.

\section{References}

1 Licata C, Pasqualin A, Freschini A, Barone G, Da Pian R. Management of associated primary cerebral neoplasms and vascular malformations: 2. Intracranial arterio-venous malformations. Acta Neurochir (Wien) 1986;83(1-2):38-46

2 Yano H, Nakayama N, Ohe N, et al. Surgical strategy in case with co-existence of malignant oligodendroglioma and arteriovenous malformation: a case report. Case Rep Clin Med 2013;2:473-478

3 Bitoh S, Hasegawa H, Kato A, Tamura K, Mabuchi E, Kobayashi Y. Meningeal neoplasms associated with cerebral vascular malformations. Surg Neurol 1987;27(5):469-475 